\title{
When fear does not serve survival: anxiety disorders viewed within a developmentally appropriate context
}

\author{
Giulia Signorini $^{1}$
}

Published online: 21 January 2019

○) Springer-Verlag GmbH Germany, part of Springer Nature 2019

Fear is one of the most self-preserving emotions among living beings, enabling them to detect, predict and react to hazards (e.g., with flight or fight responses), guaranteeing survival. On a cognitive perspective, fear-and its anticipatory version, anxiety - can be sustained by a 'better safe than sorry' principle: when facing unknown or unclear situations, we automatically tend to estimate that fearing a potential danger is more preferable than ignoring the possible existence of some risks. Thus, the costs associated with facing an unpredicted danger are considered higher than those met for worrying about it in advance. In evolutionary terms, this assumption makes perfect sense, but what happens when anxiety or fear are pervasively elicited by unrealistic, very remote or unpreventable types of threats within a given culture and context? And what happens when this occurs to human beings still in the process of building up their resilience and coping skills, still developing their representations of the world they live in as children and adolescents?

\section{When fear becomes a disorder}

According to DSM-5, the epidemiology of anxiety disorders (ADs) includes lifetime prevalence rates ranging from $0.03 \%$ (selective mutism) to $9 \%$ (specific phobia), which differ geographically and by sex with a female to male ratio of 2:0 for the majority of the respective disorders. In childhood and adolescence, ADs are the most prevalent mental disorders, with age-related dependencies, such as separation anxiety disorder/specific phobias and social phobia being more common in childhood and adolescence, respectively. Progression, persistence and comorbidities also represent

Giulia Signorini

gsignorini@fatebenefratelli.eu

1 Psychiatric Epidemiology and Evaluation Unit, IRCCS Istituto Centro San Giovanni di Dio Fatebenefratelli, Via Pilastroni 4, 25125 Brescia, Italy important aspects to consider when outlining characteristics of ADs. ADs often show homotypic and heterotypic comorbidities, among the latter especially depressive disorders (DDs). The presence of comorbidities affects individual functioning and prognosis, thus potentially complicating the clinical outcomes. As previously examined [1], the course of ADs and DDs includes, if not predicts, the exacerbation of additional disorders, with heterotypic more frequent than homotypic continuity. This explains why, if untreated, ADs (and their comorbidities) tend to persist during adulthood, representing a major challenge for public health system.

The work of Canals et al. presented in this issue [2] provides robust data on the epidemiology of ADs in Spain via screening and a 2-year follow-up of a cohort of 1514 school children. A slightly higher (11.8\%) weighted prevalence of ADs was detected as compared to other industrialized countries; the investigators also confirmed a higher heterotypic than homotypic comorbidity and identified generalized anxiety disorder (GAD) as a predictor for future depression, revealing important clinical implications in terms of necessary preventive initiatives toward childhood and adolescent mental health. On the same note, access to care for disadvantaged youths is strongly encouraged by the authors, as a low socioeconomic environment represents a risk factor for ADs. This is particularly relevant considering (1) the scarce (only one-third) request for professional help and (2) the persistence of symptoms of ADs after 2 years in more than half of the sample. Similar to most other mental disorders with a childhood or adolescent onset, ADs must be targeted by developmentally appropriate screening, prevention and treatment programs, to prevent the development of more severe clinical conditions and poorer functioning later in life.

\section{Responding to fear}

To tackle the persistence of ADs into adulthood and their overall poor prognosis, three key aspects should be pursued, 
(1) increment preventive/health promotion initiatives for children and adolescents in the general population,

(2) identify and overcome barriers to care for youths suffering from anxiety disorders and,

(3) offer them evidence-based treatments.

Preventive initiatives target both risk and protective factors playing a role in the development of a clinical condition. Among risk factors, stress exposure can strongly affect children's perception of threat and their reaction to it, often resulting in hyper-sensitization. Such exposure can happen both during acute life events as well as during ongoing chronic adversities experienced in the living context, i.e., their families. In this respect, the case of growing up with a parent suffering from a mental disorder warrants particular attention. Despite a considerable amount of literature on risks and consequences of having a mentally ill parent, only single studies have focused on the relationship between parent psychopathology and stress generation for ADs. An interesting contribution in this context has been recently provided by Allen et al. [3], whereby children with ADs, as compared to healthy controls, showed significantly more parent-related chronic adversities (such as parental conflict, parental conflict with child's teacher and with respect to the child's behaviour). This points out how preventive initiatives should not be limited to the general population, but should also target vulnerable families: this may not be accomplished by single community or school-based prevention programs, but instead potentially requires close interaction with further levels of care, i.e., adult mental health services for treatment of parents. At the protective level, promotion of healthy life styles should also be targeted: results from the Saving and Empowering Youth Lives in Europe (SAYLE) study [4] have recently provided strong support to the idea that physical activity (at least $60 \mathrm{~min}$ per day) and sport participation do effectively contribute to lowering levels of anxiety and depression in youths.

Children and adolescent families may face several barriers to care, which range from inadequately trained or unavailable therapists, too long waiting lists, paucity of services in their living area, stigma and prejudice toward mental illness. Moreover, in the case of ADs, (i.e., social phobia), symptoms can be underestimated by adults and misrecognized as, for example, introversion and shy personality, hence often delaying a prompt access to care. Regardless of whether the barriers belong more to an institutional or individual/cultural type, they may prevent youth from getting diagnosed and treated in a timely and proper manner. This can happen even if services are organized and functioning as intended according to type and severity of problems [5].

A possible solution to overcome such barriers has been tested in the study of David et al., presented in this issue [6], i.e., using the fascinating idea of therapeutic games, offers an interesting potential for the implementation of new technologies in the healthcare system. The idea is to provide youths with easily accessible and appealing training programs aimed at increasing their resilience and coping skills by focusing on trans-diagnostic features such as emotion regulation strategies. Emotion regulation has been, in fact, proposed as an important maintaining mechanism in ADs [7]: compared to healthy peers, children with social anxiety and mixed ADs show less adaptive and more maladaptive emotion regulation strategies. Treatment programs offering focused training of such abilities may therefore represent a promising, overarching and complementary approach in youth mental health care. Needless to say, this idea can be adopted also for health promotion/prevention programs.

Finally, with regard to interventions, to act in a proper and effective manner, it is imperative to adopt treatment protocols that have been shown to be evidence-based and sustainable (cost-effective and acceptable). Yang et al.'s meta-analysis, included in this issue [8], focusses on randomized controlled trials (RCTs) for children and adolescents with social anxiety disorder or social phobia. The authors analyzed 17 RCTs and concluded that, compared to control conditions, psychological interventions may effectively help in reducing psychopathology (including comorbid depressive symptoms) and improve well-being and functioning. Insights on which type and format of intervention (group, individual, family, internet based) should be adopted for which specific clinical goal are also provided.

\section{Conclusions}

Derived from the Latin word 'angere', 'anxiety' can be translated into 'narrowing'. This really depicts the idea of suffering from a mental disorder whereby fear and worrying limit individual freedom of expression and the secure transition towards new challenges and coping with adversities, which all represent very developmental achievements.

While taking care of patients with such clinical conditions, professionals are encouraged to balance validation of the underlying fear circuit by promoting psycho-educative and non-stigmatizing approaches toward fear reactions (starting from the community/primary care level), with change of individual responses to fear, by adopting available evidence-based and cost-effective treatments.

\section{References}

1. Ranoyen I, Lydersen S, Larose TL, Weidle B, Skokauskas N, Thomsen PH, Wallander J, Indredavik MS (2018) Developmental course of anxiety and depression from adolescence to young adulthood in a prospective Norwegian clinical cohort. Eur Child 
Adolesc Psychiatry 27:1413-1423. https://doi.org/10.1007/s0078 7-018-1139-7

2. Canals J, Voltas N, Hernández-Martínez C et al (2018) Prevalence of DSM-5 anxiety disorders, comorbidity, and persistence of symptoms in Spanish early adolescents. Eur Child Adolesc Psychiatry. https://doi.org/10.1007/s00787-018-1207-z Epub July 28, 2018

3. Allen JL, Sandberg S, Chhoa CY, Fearn T, Rapee RM (2018) Parent-dependent stressors and the onset of anxiety disorders in children: links with parental psychopathology. Eur Child Adolesc Psychiatry 27(2):221-231. https://doi.org/10.1007/s00787-0171038-3 Epub Aug 8, 2017

4. McMahon EM, Corcoran P, O'Regan G et al (2017) Physical activity in European adolescents and associations with anxiety, depression and well-being. Eur Child Adolesc Psychiatry 26(1):111-122. https://doi.org/10.1007/s00787-016-0875-9 Epub Jun 9, 2016

5. Nanninga M, Jansen D, Knorth EJ, Reijneveld SA (2018) Enrolment of children in psychosocial care: problems upon entry, care received, and outcomes achieved. Eur Child Adolesc Psychiatry 27:625-635. https://doi.org/10.1007/s00787-017-1048-1

6. David OA, Cardos RAI, Matu S (2018) Is REThink therapeutic game effective in preventing emotional disorders in children and adolescents? Outcomes of a randomized clinical trial. Eur Child Adolesc Psychiatry. https://doi.org/10.1007/s00787-018-1192-2 Epub July 10, 2018

7. Keil V, Asbrand J, Tuschen-Caffier B, Schmitz J (2017) Children with social anxiety and other anxiety disorders show similar deficits in habitual emotional regulation: evidence for a transdiagnostic phenomenon. Eur Child Adolesc Psychiatry 26(7):749-757. https://doi.org/10.1007/s00787-017-0942-x Epub Jan 11, 2017

8. Yang L, Zhou X, Pu J, Liu L et al (2018) Efficacy and acceptability of psychological interventions for social anxiety disorder in children and adolescents: a meta-analysis of randomized controlled trials. Eur Child Adolesc Psychiatry. https://doi.org/10.1007/ s00787-018-1182-4 Epub June 15, 2018 